\title{
A Search In Scientific Medicine
}

\author{
Dr. Ikam-Anthony Igbojekwe \\ Geriatric Clinician and Medical Lipid Biochemist, Member Royal Society of Biology, UK \\ DOI: 10.29322/IJSRP.11.07.2021.p11552 \\ http://dx.doi.org/10.29322/IJSRP.11.07.2021.p11552
}

\begin{abstract}
This search was not specifically started to find a solution to the treatment of Human immuno deficiency viral infections (AIDS). Notwithstanding, it may be a coincidence following several hypothesis and theories both nascent and retrospective so to say. Science is becoming more and more complex as living organisms continues to evolve and we enter the next millennium which is shaking.
\end{abstract}

The history and/or origin of this search could be dated since 1982 while comparing the differences between living and nonliving thing. It is highly a complex one and outcome may be numerous. With the support of other medical professionals (Biochemists, Pharmacologist, Microbiologist, Pathologist, Physicians and Surgeons).

\section{INTRODUCTION}

The tips:

a) DNA can be made from RNA (Temin and David Baltimore 1970). This was demonstrated in several avain and murine retroviruses by the enzyme reverse transcriptase. The enzymatic activities of reverse transcriptase include; coping RNA molecule to yield a double stranded DNA-RNA using a primer and joining deoxyribonucleic acid-5-triphosphate in 3, 5' linkage. It can also copy a primed single stranded DNA to form double stranded DNA. It degrades RNA in DNA-RNA HYBRID (Rnase $\mathrm{H}$ activity). Appropriate primer will potentiate the single strand to be converted to double stranded DNA.

b) There can be statement of frustration in science, the position of atomic particles may not be known exactly, when it changes momentum and why. In uncertainty principle (Werner Heisenberg 1927) there is statistical probability of finding an electron at any particular point in time in a given volume of space. The power of quantum mechanics is as great in the realm of sub atomic particles as Newtonian mechanics is for larger bodies. Hence, the uncertainty principle can be applied to all objects, living and non-living things.

c) There was chemistry before life (human evolutionary biology). A substance is common to all cancer cells. This makes them differ from the actual natural cells/tissues in metabolism, although the substance does not interfere with the cancer cells competing with the natural body cells and tissues. This substance is being stimulated most as blood supply to the cancer cells increase. Tissues of the same embryological origin have their own particular substance. However, there is a relationship between the activities of the cancer cells and the pathogenic effect of some viral infections. The effect of glycoproteins.

\section{CORONAVIRUS PANDEMIC}

\section{BIOCHEMICAL PERSPECTIVE}

Presence of genome, natural selection and evolution are the basic characteristics that qualifies virus as a living matter, but not exhaustive and unreliable.

In this context virus is a complex molecular assembly that includes nucleic acid protein, lipids and enzyme in which a substance must stimulate its biochemical reactions in the living host. Externally in the ecosystem the substance is not activated for viral self - replication.

Internally, within the host (internal ecosystem) the substance is activated by cascade of mechanism. The corona-virus is a single stranded RNA and unsegmented genome. It can be isolated from upper respiratory and/or gastro intestinal tract.

The route of entry for the viral particle seems to depend on the complete cell unit of the host. The lipid bi layer interface in the gastrointestinal and respiratory tract harbor the molecules that stimulate the biochemical reactions which facilities the entry of viral genome in the host. The role of transcription factors in viral reproductive cycle is equally a matter of concern.

In other words the biochemical reaction between the viral envelop and cell membrane of the host must be stimulated by biochemical substance. There it uncoats, get incorporated into the cell metabolic processes before replication.

The consequent viral and host genome interaction provoke the clinical pathologic conditions associated with viruses.

In the tropics where protozoa infections predominate (eg. Plamodiasis, and Amoebiasis) the corona-viral genome is likely to infect the protozoa. In which case the viral load may be reduced for clinical manifestation in humans especially as they pass through the lymphatic glands.

The viral and protozoal agents interact with the immune response (cytokine). Antigen -antibody reactions.

Safe antigenic vaccine is very important to avoid emergence of very dangerous strains; which may lead to extinction of 
homosapiens (human) from the universe. Proactive measure necessary.

\section{POINT OF CONCERN}

Considering the biochemical relationship between cancer and viral agents immuno- modulatory effects of drugs should be put into study to help vaccines in the eradication of COVID-19. Known Immunomodulatory like Ergamisol (Jansen Pharmaceuticals) can provide for the necessary research for clinical trials. required.

However, disruption of biochemical assembly is highly

\section{AUTHORS}

Dr. Ikam Anthony Igbojekwe, MBBCH, FLIC, MRSB (UK), Geriatrics/Lipid Biochemistry 